\title{
Spatial Resolution Properties of Penalized-Likelihood Image Reconstruction: Space-Invariant Tomographs
}

\author{
Jeffrey A. Fessler, Member, IEEE, and W. Leslie Rogers, Member, IEEE
}

\begin{abstract}
This paper examines the spatial resolution properties of penalized-likelihood image reconstruction methods by analyzing the local impulse response. The analysis shows that standard regularization penalties induce space-variant local impulse response functions, even for space-invariant tomographic systems. Paradoxically, for emission image reconstruction, the local resolution is generally poorest in high-count regions. We show that the linearized local impulse response induced by quadratic roughness penalties depends on the object only through its projections. This analysis leads naturally to a modified regularization penalty that yields reconstructed images with nearly uniform resolution. The modified penalty also provides a very practical method for choosing the regularization parameter to obtain a specified resolution in images reconstructed by penalized-likelihood methods.
\end{abstract}

\section{INTRODUCTION}

O $\mathrm{T}$ TATISTICAL methods for image reconstruction can proide improved spatial resolution and noise properties over conventional filtered backprojection (FBP) methods. However, iterative methods based solely on maximum-likelihood criteria produce images that become unacceptably noisy as the iterations proceed. Methods for reducing this noise include i) stopping the iteration before the images become too noisy (long before convergence) [1], ii) iterating until convergence and then post-smoothing the image [2], iii) using smooth basis functions [3], and iv) replacing the maximum-likelihood criterion with a penalized-likelihood (or maximum a posteriori) objective function that includes a roughness penalty to encourage image smoothness [4].

Penalized-likelihood approaches for reducing noise have two important advantages over alternatives such as stopping rules and sieves. First, the penalty function improves the conditioning of the problem, so certain iterative algorithms converge very quickly. Second, one can choose penalty functions that control desired properties of the reconstructed images, such as preserving edges [4] or incorporating anatomical side information [5], [6]. In contrast, the smoothness that one obtains through stopping rules is limited by the characteristics of the iterative algorithm. A possible disadvantage of penalizedlikelihood methods has been the absence of an intuitive method for choosing the value of the regularization parameter, even for simple quadratic penalties. One contribution of this paper is a new object-independent method for specifying the

Manuscript received September 2, 1995; revised January 11, 1996. This work was supported in part by the NIH under Grants CA-60711 and CA54362. The associate editor coordinating the review of this manuscript and approving it for publication was Prof. Ken D. Sauer.

The authors are with the University of Michigan, Ann Arbor, MI 481092122 USA (e-mail: fessler@umich.edu).

Publisher Item Identifier S 1057-7149(96)06032-0. regularization parameter in terms of the desired resolution of the reconstructed image.

This paper describes another possibly undesirable property of penalized-likelihood image reconstruction methods that has not been previously documented (except in [7], to our knowledge), and then proposes a solution to the problem. Through analysis and empirical results, we demonstrate that when one uses standard space-invariant roughness penalties, the reconstructed images have object-dependent nonuniform spatial resolution, even for space-invariant tomographic systems. For emission imaging, the resolution is generally poorest in high-count regions, which is the opposite of what one might expect or prefer. In Section V, we propose a new modified space-variant roughness penalty that yields images with nearly uniform resolution. Based on our analysis, one could extend the method to provide other resolution characteristics, such as higher resolution in high-count regions, in a manner similar to methods for space-varying regularization [8], [9]; however, in this paper we focus on the goal of providing uniform resolution.

This paper is somewhat in the spirit of previous studies that used the local impulse response [10]-[14] or an effective local Gaussian resolution [15] to quantify the resolution properties of the unregularized maximum-likelihood expectation-maximization (MLEM) algorithm for emission tomography. However, there is an important difference in our approach: Since the MLEM algorithm is rarely iterated until convergence, previous studies examined the spatial resolution properties of MLEM as a function of iteration. In contrast, since there are now fast and globally convergent algorithms for maximizing both penalized-likelihood [16]-[19] and penalized weighted least squares [20]-[22] objective functions, rather than studying the properties of the algorithms as a function of iteration, we study directly the properties of the estimator as specified by the objective function (Sections II and III). This simplifies the practical use and interpretation of our analysis, since the specifics of the iterative algorithm are unimportant (provided one uses a globally convergent method). Our main results (14) and (16) should therefore be applicable to a broad range of inverse problems. (Although we focus on image reconstruction, most of the issues also pertain to quantumlimited image restoration.)

In conventional FBP image reconstruction, one controls the tradeoff between resolution and noise by adjusting the cutoff frequency $f_{c}$ of a filter. Since $f_{c}$ has units of inverse length, there is an intuitive (and object-independent) relationship between $f_{c}$ and the spatial resolution of the reconstructed 
image. For idealized tomographs, one can use the Hankel transform to compute the point spread function (PSF) as a function of $f_{c}$ [23]. But for real systems, one usually determines the (monotonic) relationship between $f_{c}$ and the full-width half-maximum (FWHM) of the PSF through the following empirical approach. First, acquire a sinogram using a point or line source, possibly at several locations within the scanner. Then pick a filter type (e.g., Hanning) and reconstruct images for several different values of $f_{c}$. Finally, compute the FWHM of the PSF for each case, and record a table of $\left(f_{c}\right.$, FWHM) value pairs. In subsequent studies, one typically chooses the desired resolution (FWHM) by experience or by visually observing the resolution-noise tradeoff, and then obtains the appropriate $f_{c}$ from the table. One needs to perform this tabulation only once for a given scanner, since FBP is linear (and hence its resolution properties are object independent).

In contrast, in penalized-likelihood image reconstruction, a regularization parameter $\beta$ controls the tradeoff between resolution and noise, but the units of $\beta$ are at best opaquely related to spatial resolution. Therefore, it is not obvious how to specify the regularization parameter. As a further complication, one finds that for a fixed $\beta$, the reconstructed spatial resolution varies between subjects, and even within the same subject (Section IV). One could choose $\beta$ using statistical criteria such as minimum mean-squared error [24], [25]. However, meansquared error is composed equally of both bias (resolution) and variance (noise), whereas those two contributions usually have unequal importance in medical imaging, particularly when images are to be interpreted visually. Furthermore, datadriven methods for choosing $\beta$ can be unstable in imaging problems [26]. Many other alternatives have been proposed, e.g., [27] and [28], most of which have again been assessed with respect to mean-squared error. One practical contribution of this paper is that we develop a method for normalizing the penalty function such that the object-dependent component of $\beta$ is nearly eliminated. This allows one to build an objectindependent table relating $\beta$ to spatial resolution (FWHM) for a given tomographic system, so that one can select $\beta$ to achieve a consistent specified resolution within planes, between planes, and even between subjects. The task of choosing the "optimal" resolution is left to the user, just as the "optimal" cutoff frequency (and filter) for FBP are determined by different criteria in different contexts.

Nonuniform resolution properties are not unique to penalized-likelihood methods. The MLEM algorithm for emission tomography also exhibits resolution variation and asymmetry [11], [29]. An advantage of the penalizedlikelihood approach is that one can modify the penalty to overcome the resolution nonuniformity (Sections V, VI, and VII), whereas it is not obvious how to modify MLEM to achieve uniform resolution.

Positron emission tomography (PET) and single photon emission computed tomography (SPECT) systems usually have intrinsically nonuniform spatial resolution [30] (although PET systems are usually nearly space invariant near the center of the scanner [30]). In this paper, our simulations focus on an idealized PET system that is essentially space invariant, except perhaps for the effects of discretizing the Radon transform. Thus, the resolution nonuniformities we report are due solely to the interaction between the log-likelihood and the penalty terms of the objective function, and not due to the system response. In future work, we hope to study the effects of penalty functions in systems with intrinsically space-variant resolution.

This paper is condensed from [31]. In [31], we also analyze a continuous idealization of penalized least-squares image reconstruction.

\section{LOCAL IMPULSE RESPONSE}

Let $Y=\left[Y_{1}, \cdots, Y_{N}\right]^{\prime}$ denote a random measurement vector (e.g. a noisy sinogram) with density function $f(y ; \theta)$, where $\theta=\left[\theta_{1}, \cdots, \theta_{p}\right]^{\prime}$ is an unknown parameter in a $p$ dimensional parameter space $\Theta$, and 'denotes vector transpose. In imaging problems, $\theta$ typically denotes image pixel values in lexicographic ordering and $\Theta=\left\{\theta: \theta_{j} \geq 0, j=\right.$ $1, \cdots, p\}$. Given a particular realization $Y=y$, an estimator of the form $\hat{\theta}=\hat{\theta}(y)$ has mean

$$
\mu(\theta)=E_{\theta}[\hat{\theta}(Y)]=\int \hat{\theta}(y) f(y ; \theta) d y .
$$

For linear and space-invariant problems, one can characterize the properties of $\mu$ either in the spatial domain by specifying the (global) impulse response, or in the spectral domain by specifying the frequency response (Fourier transform of the impulse response), as in [31].

Spectral methods are generally inapplicable to nonlinear estimators for which the impulse response is space variant. For nonlinear estimators, one can analyze the local impulse response (cf [11]). For an estimator with mean $\mu(\theta)$, we define the local impulse response of the $j$ th parameter (pixel) to be ${ }^{1}$

$$
\begin{aligned}
l^{j}(\theta) & =\lim _{\delta \rightarrow 0} \frac{\mu\left(\theta+\delta e^{j}\right)-\mu(\theta)}{\delta} \\
& =\frac{\partial}{\partial \theta_{j}} \mu(\theta), \quad j=1, \cdots, p,
\end{aligned}
$$

where $e^{j}$ is the $j$ th unit vector of length $p$. This impulse response is local in two different senses. First, it is a function of the index $j$, reflecting the space-variant nature of nonlinear estimation. Second, it depends on the location in the parameter space $\Theta$ through the argument $\theta$, reflecting the nonlinear object dependence. The local impulse response also depends on the measurement distribution through (1). Thus, the local impulse response characterizes the object, system, and estimator-dependent properties. The local impulse response measures the change in the mean reconstructed image due to perturbation of a particular pixel in the noiseless object. ${ }^{2}$

To confirm that (2) is a natural generalization of the usual definition of impulse response, consider an estimator whose

\footnotetext{
${ }^{1}$ We restrict our discussion to estimators where the above limit is well defined. The reader is cautioned that nonconvex penalties can lead to estimates that are discontinuous functions of the data [32]. We focus here on wellbehaved convex penalties.

${ }^{2}$ Because of this interpretation, we use the term point spread function (PSF) synonymously with local impulse response, even though this stretches the usual meaning of PSF.
} 

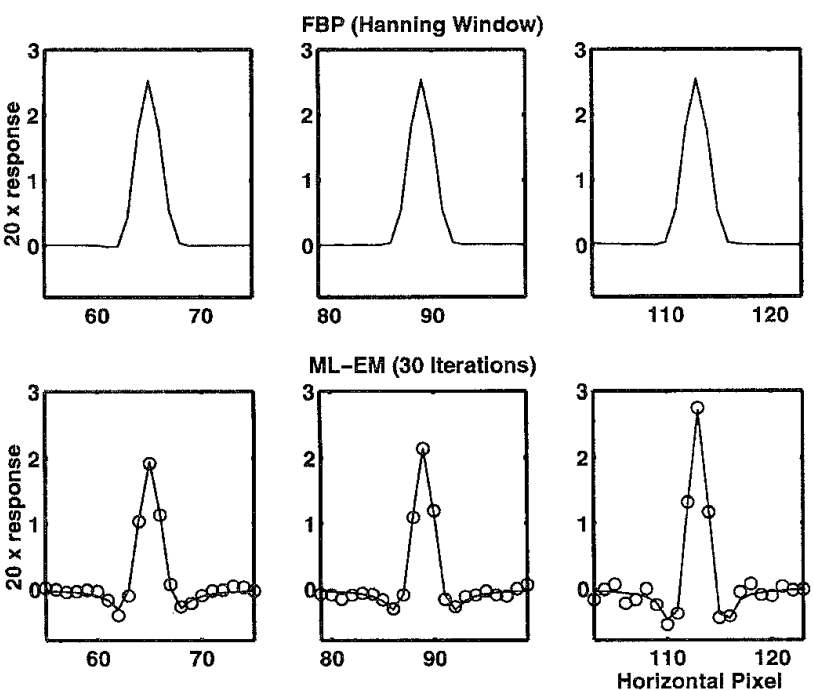

Fig. 1. Horizontal profiles through the local impulse response functions of FBP with a Hanning window (top) and of the MLEM algorithm at 30 iterations (bottom), for three pixels located along the horizontal midline of an elliptical object. Solid line: computed using the linearized approximation (10). Circles: computed using the unbiased estimator (6) from $M=2000$ realizations.

mean is linear in $\theta: \mu(\theta)=L \theta$. Then the conventional definition of impulse response is $\mu\left(e^{j}\right)$, which is the $j$ th column of $\boldsymbol{L}$. Evaluating (2), one finds that $l^{j}$ is also the $j$ th column of $L$. (If, in addition, $L$ is a circulant matrix, then the impulse response is space invariant, and $L$ corresponds to a convolution [33].) Also note that $\mu(\theta)=\theta$ for unbiased estimators, in which case $l^{j}=e^{j}$. Penalized-likelihood estimators are always biased, so local impulse responses will typically be bump-like functions rather than the ideal impulse $e^{j}$ (e.g., Fig. 1).

As a specific example, consider the following penalized weighted least-squares estimator [21]:

$$
\hat{\theta}=\hat{\theta}(y)=\arg \min _{\theta}(y-\boldsymbol{A} \theta)^{\prime} \boldsymbol{W}(y-\boldsymbol{A} \theta)+\beta \theta^{\prime} \boldsymbol{R} \theta,
$$

where $\boldsymbol{W}$ and $\boldsymbol{R}$ are symmetric nonnegative definite matrices for which the null spaces of $\boldsymbol{R}$ and $\boldsymbol{W} \boldsymbol{A}$ are disjoint. For a fixed $W$, this estimator is linear in $y$ :

$$
\hat{\theta}(y)=\left[\boldsymbol{A}^{\prime} \boldsymbol{W} \boldsymbol{A}+\beta \boldsymbol{R}\right]^{-1} \boldsymbol{A}^{\prime} \boldsymbol{W} y
$$

and, assuming $E_{\theta}[Y]=A \theta$, one can evaluate (2) to show

$$
l^{j}=\left[\boldsymbol{A}^{\prime} \boldsymbol{W} \boldsymbol{A}+\beta \boldsymbol{R}\right]^{-1} \boldsymbol{A}^{\prime} \boldsymbol{W} \boldsymbol{A} e^{j}
$$

For such linear estimators, the local impulse response is independent of $\theta$. As we show in Section III, the local impulse responses of the nonlinear penalized-likelihood estimators for image reconstruction have approximately the same form as (3), except that $\boldsymbol{W}$ and $\boldsymbol{R}$ may depend on $\theta$.

There are at least three reasons to study the local impulse response. The first reason is simply to understand the resolution properties of penalized-likelihood estimators. The second reason is that the local impulse response allows one to quantify local resolution, which in turn allows one to choose the smoothing parameter $\beta$ sensibly. The third reason is that comprehension of the resolution properties enables the design of better penalty functions. In particular, we show how to modify the standard regularization penalty to achieve nearly uniform resolution.

\section{A. Brute Force Evaluation of Local Impulse Response}

Unlike the simple penalized weighted least-squares estimator described above, most estimators $\hat{\theta}(y)$ do not have an explicit analytical form. When there is no explicit form for $\hat{\theta}(y)$, there is usually no explicit form for the estimator mean $\mu(\theta)$ either. Thus, it would at first appear that to investigate the local impulse response of a nonlinear estimator of interest, one must resort to a numerical approach based on (1) and (2), replacing the expectation in (2) by the sample mean in a computer simulation. The following recipe illustrates this brute-force approach.

- Select an object $\theta$ of interest and generate multiple realizations $\left\{y^{(m)}\right\}_{m=1}^{M}$ of noisy measurements according to the density $f(y ; \theta)$.

- Apply the estimator of interest to each of the measurement realizations to obtain estimates $\left\{\hat{\theta}\left(y^{(m)}\right)\right\}_{m=1}^{M}$.

- Estimate the estimator mean using the sample mean

$$
\hat{\mu}(\theta)=\frac{1}{M} \sum_{m=1}^{M} \hat{\theta}\left(y^{(m)}\right) .
$$

- Choose a pixel $j$ of interest and small value $\delta$, and generate a second set of noisy measurements according to the density $f\left(y ; \theta+\delta e^{j}\right)$.

- Apply the estimator to the second set of noisy measurements and compute the sample mean to obtain an estimate $\hat{\mu}\left(\theta+\delta e^{j}\right)$.

- Estimate the local impulse response

$$
l^{j}(\theta) \approx \frac{\hat{\mu}\left(\theta+\delta e^{j}\right)-\hat{\mu}(\theta)}{\delta} .
$$

By taking $\delta$ sufficiently small and $M$ sufficiently large, one can obtain arbitrarily accurate estimates of the local impulse response.

\section{B. Unbiased Estimator for Local Impulse Response}

If one wants to evaluate the local impulse response for pixels $j_{1}, \cdots, j_{L}$ of interest, the above procedure requires $(L+$ 1) $M$ image reconstructions. The following method [34]-[36] reduces the computation to only $M$ image reconstructions. Note that from (2),

$$
\begin{aligned}
l^{j}(\theta) & =\frac{\partial}{\partial \theta_{j}} \mu(\theta)=\frac{\partial}{\partial \theta_{j}} E_{\theta}[\hat{\theta}(Y)]=\frac{\partial}{\partial \theta_{j}} \int \hat{\theta}(y) f(y ; \theta) d y \\
& =E_{\theta}\left[\hat{\theta}(Y) \frac{\partial}{\partial \theta_{j}} \log f(Y ; \theta)\right] .
\end{aligned}
$$

Thus one can show [35], [36] that

$$
\hat{l}^{j}(\theta)=\frac{1}{M-1} \sum_{m=1}^{M}\left(\hat{\theta}\left(y^{(m)}\right)-\hat{\mu}(\theta)\right) \frac{\partial \log f\left(y^{(m)} ; \theta\right)}{\partial \theta_{j}}
$$


is an unbiased estimator for $l^{j}(\theta)$, where $\hat{\mu}(\theta)$ was defined in (4). Once one performs the $M$ reconstructions $\left\{\hat{\theta}\left(y^{(m)}\right)\right\}_{m=1}^{M}$, then one can estimate the local impulse response $\hat{l}^{j}(\theta)$ for many pixels with little additional effort.

By taking $M$ sufficiently large, one can obtain arbitrarily accurate estimates of the local impulse response. Unfortunately, $M$ may need to be very large for sufficient accuracy. Often, we would gladly accept an approximation to the local impulse response if we could avoid performing extensive numerical simulations. The remainder of this paper is devoted to approximations suitable for likelihood-based estimators in tomography.

\section{Linearized Local Impulse Response}

In the context of emission tomography, several investigators [13], [14], [37], [38] have observed that the ensemble mean of a likelihood-based estimator is approximately equal to the value that one obtains by applying the estimator to noiseless data

$$
\mu(\theta)=E_{\theta}[\hat{\theta}(Y)] \approx \hat{\theta}(\bar{Y}(\theta)) \triangleq \breve{\theta} .
$$

Here

$$
\bar{Y}(\theta)=E_{\theta}[Y]=\int y f(y ; \theta) d y
$$

denotes the mean of the measurement vector, and $\breve{\theta}$ denotes the value of the estimator when given noiseless data $\bar{Y}(\theta)$. This approximation is equivalent to assuming that the estimator is locally linear. Let $\nabla_{y}=\left[\left(\partial / \partial y_{1}\right) \cdots\left(\partial / \partial y_{N}\right)\right]$ and consider the first-order Taylor expansion of $\hat{\theta}(Y)$ about $\bar{Y}(\theta)$ :

$$
\hat{\theta}(y) \approx \hat{\theta}(\bar{Y}(\theta))+\nabla_{y} \hat{\theta}(\bar{Y}(\theta)) \cdot(Y-\bar{Y}(\theta)) .
$$

Taking the expectation of both sides yields (7). The remainder of this paper uses this local linearity approximation.

Substituting (7) into (2) yields the following definition of the linearized local impulse response:

$$
\begin{aligned}
l^{j}(\theta) & =\lim _{\delta \rightarrow 0} \frac{\hat{\theta}\left(\bar{Y}\left(\theta+\delta e^{j}\right)\right)-\hat{\theta}(\bar{Y}(\theta))}{\delta} \\
& =\frac{\partial}{\partial \theta_{j}} \hat{\theta}(\bar{Y}(\theta)) .
\end{aligned}
$$

Since we focus on this form in the remainder of this paper, for brevity we usually omit the adjective "linearized."

The form of (9) leads to a much simpler recipe for numerically evaluating the local impulse response.

- Select an object $\theta$ of interest, a pixel $j$ of interest, and a small value $\delta$. Generate two noiseless measurements vectors: $\bar{Y}(\theta)$ and $\bar{Y}\left(\theta+\delta e^{j}\right)$.

- Apply the estimator of interest to each of the two noiseless measurements, obtaining estimates $\hat{\theta}(\bar{Y}(\theta))$ and $\hat{\theta}(\bar{Y}(\theta+$ $\left.\left.\delta e^{j}\right)\right)$.

- Estimate the local impulse response

$$
l^{j}(\theta) \approx \frac{\hat{\theta}\left(\bar{Y}\left(\theta+\delta e^{j}\right)\right)-\hat{\theta}(\bar{Y}(\theta))}{\delta} .
$$

By taking $\delta$ sufficiently small, one can obtain very accurate estimates of the linearized local impulse response. If $\hat{\theta}$ is linear in $y$, then (10) is exact, of course.

To illustrate this method, Fig. 1 shows a profile through several local impulse response functions of FBP and of the emission MLEM algorithm [39] (stopped at 30 iterations, well before convergence). The object $\theta$ was a uniform ellipse of activity within a uniform elliptical attenuator (see [31] for details). Despite the fact that the elliptical object has uniform activity, the resolution of the nonlinear MLEM estimator is clearly nonuniform, whereas the FBP resolution is uniform, since the smoothing provided by the Hanning window is space invariant. Using a similar perturbation approach applied to both the noiseless mean of the data $\bar{Y}(\theta)$ and to a single noisy realization $Y$, Stamos et al. [10] reported strongly object-dependent point response functions for the algebraic reconstruction technique (ART) and MLEM algorithms.

Several investigators have used this easily implemented empirical approach to study the properties of maximumlikelihood estimators in emission tomography. However, being empirical, it fails to reveal general estimator properties. An analytical expression for the linearized local impulse response would facilitate understanding general properties of image reconstruction methods. The next section derives an analytical expression for the local impulse response of implicitly defined estimators.

\section{ANALYSis OF LOCAL IMPULSE RESPONSE FOR IMPLICITLY DEFINED ESTIMATORS}

Many estimators in tomography are defined implicitly as the maximizer of some objective function:

$$
\hat{\theta}=\hat{\theta}(y)=\arg \max _{\theta \in \Theta} \Phi(\theta, y) .
$$

We assume $\Phi$ has a unique global maximum, so that $\hat{\theta}(y)$ is well defined. There is often no analytical form for such estimators; hence, the ubiquitous use of iterative algorithms for performing the required maximization. Fortunately, the linearized local impulse response (9) depends only on the partial derivatives of the implicitly defined estimator $\hat{\theta}(y)$. As discussed in [38], even though $\hat{\theta}(y)$ itself is unknown, one can determine its partial derivatives using the implicit function theorem and the chain rule. Disregarding the nonnegativity constraint, ${ }^{3}$ the maximizer of $\Phi$ satisfies

$$
\left.\frac{\partial}{\partial \theta_{j}} \Phi(\theta, y)\right|_{\theta=\hat{\theta}(y)}=0, \quad j=1, \cdots, p
$$

for any $y$. In vector notation

$$
\nabla^{10} \Phi(\hat{\theta}(y), y)=0 \quad \forall y,
$$

where $\nabla^{10}=\left[\left(\partial / \partial \theta_{1}\right) \cdots\left(\partial / \partial \theta_{p}\right)\right]$ is the row gradient operator (with respect to the first argument of $\Phi$ ). Now differentiate again with respect to $y$ using the chain rule

$$
\nabla^{20} \Phi(\hat{\theta}(y), y) \nabla_{y} \hat{\theta}(y)+\nabla^{11} \Phi(\hat{\theta}(y), y)=0
$$

${ }^{3}$ Although it appears we are assuming that (12) holds for any $y$, from (9) one sees we really only need (12) to hold near the case $y=\bar{Y}(\theta)$, i.e., the noiseless case. The nonnegativity constraint is often largely inactive for noiseless data, so (12) is a reasonable assumption. 
where the $(j, k)$ th element of $\nabla^{20}$ is $\partial^{2} / \partial \theta_{j} \partial \theta_{k}$ and the $(j, i)$ th element of $\nabla^{11}$ is $\partial^{2} / \partial \theta_{j} \partial y_{i}$. For simplicity, we drop the dependence of $\bar{Y}$ on $\theta$ except where explicitly needed. Assuming that $-\nabla^{20} \Phi(\breve{\theta}, \bar{Y})$ is positive definite, substitute $y=\bar{Y}$ into (13) and solve for the partial derivatives of $\hat{\theta}(\bar{Y}(\theta))$ as follows:

$$
\nabla_{y} \hat{\theta}(\bar{Y}(\theta))=\left[-\nabla^{20} \Phi(\check{\theta}, \bar{Y})\right]^{-1} \nabla^{11} \Phi(\check{\theta}, \bar{Y}) .
$$

Combining with the chain rule applied to (9), we have

$$
\begin{aligned}
l^{j}(\theta) & =\frac{\partial}{\partial \theta_{j}} \hat{\theta}(\bar{Y}(\theta))=\nabla_{y} \hat{\theta}(\bar{Y}(\theta)) \frac{\partial}{\partial \theta_{j}} \bar{Y}(\theta) \\
& =\left[-\nabla^{20} \Phi(\breve{\theta}, \bar{Y})\right]^{-1} \nabla^{11} \Phi(\breve{\theta}, \bar{Y}) \frac{\partial}{\partial \theta_{j}} \bar{Y}(\theta) .
\end{aligned}
$$

This equality expresses the local impulse response solely in terms of the partial derivatives of the objective function and the measurement mean, i.e., we have eliminated the dependence on the implicitly defined estimator $\hat{\theta}(y)$.

\section{A. Penalized-Likelihood Estimators}

In the remainder of this paper, we focus on penalizedlikelihood objective functions $\Phi$ of the form:

$$
\Phi(\theta, y)=L(\theta, y)-\beta R(\theta)
$$

where $L(\theta, y)=\log f(y ; \theta)$ denotes the log-likelihood, $R(\theta)$ is a roughness penalty function, and $\beta$ is a nonnegative regularization parameter that controls the influence of the penalty, and hence the tradeoff between resolution and noise.

Define $R(\theta)=\nabla^{2} R(\theta)$ to be the Hessian of the penalty, and note that $\nabla^{11} R=0$. For penalized-likelihood estimators of the form (15) we have from (14) the following expression for the local impulse response: ${ }^{4}$

$$
l^{j}(\theta)=\left[-\nabla^{20} L(\breve{\theta}, \bar{Y})+\beta \boldsymbol{R}(\breve{\theta})\right]^{-1} \nabla^{11} L(\breve{\theta}, \bar{Y}) \frac{\partial}{\partial \theta_{j}} \bar{Y}(\theta) .
$$

This expression should be useful for investigating estimators in a variety of imaging problems. Next we evaluate expression (16) for Poisson distributed measurements, which will be the focus of the remainder of this paper.

\section{B. Poisson Statistics}

Both emission and transmission tomographic systems yield independent measurements with Poisson statistics; the primary difference is in the form of their assumed measurement means $\bar{Y}(\theta)$. In both cases, the assumed $\log$-likelihood has the form

$$
L(\theta, y)=\sum_{i} y_{i} \log \bar{Y}_{i}(\theta)-\bar{Y}_{i}(\theta)
$$

neglecting constants independent of $\theta$. In this paper, we focus on emission tomography; we derive parallel results for the transmission case in [31].

\footnotetext{
${ }^{4}$ We consider the class of objectives $\Phi$ for which the Hessian $-\nabla^{20} L(\breve{\theta}, \bar{Y})+\beta \boldsymbol{R}(\breve{\theta})$ is positive definite; i.e., $\Phi(\theta, y)$ is at least locally strictly concave near the noiseless case $(\breve{\theta}, \bar{Y}(\theta))$.
}

For emission tomography [39], $\theta_{j}$ denotes the radioisotope concentration in the $j$ th voxel, and the measurement mean is linear in $\theta$ :

$$
\bar{Y}_{i}(\theta)=\sum_{j=1}^{p} a_{i j} \theta_{j}+r_{i}
$$

The $\left\{a_{i j}\right\}$ are nonnegative constants that characterize the tomographic system, and the $\left\{r_{i}\right\}$ are nonnegative constants that represent the mean contribution of background events (random coincidences, scatter, etc.). Simple calculations [31] using (17) show that

$$
\begin{gathered}
-\nabla^{20} L(\theta, y)=\boldsymbol{A}^{\prime} D\left[\frac{y_{i}}{\bar{Y}_{i}^{2}(\theta)}\right] \boldsymbol{A} \\
\nabla^{11} L(\theta, y)=\boldsymbol{A}^{\prime} D\left[\frac{1}{\overline{\bar{Y}_{i}}(\theta)}\right],
\end{gathered}
$$

where $\boldsymbol{A}=\left\{a_{i j}\right\}$ is an $N \times p$ sparse matrix and $D\left[u_{i}\right]$ denotes an $N \times N$ diagonal matrix with diagonal entries $u_{1}, \cdots, u_{N}$. Noting that $\left(\partial / \partial \theta_{j}\right) \bar{Y}(\theta)=A e^{j}$ and substituting into (16) yields the local impulse response

$$
l^{j}(\theta)=\left[\boldsymbol{A}^{\prime} D\left[\frac{\bar{Y}_{i}(\theta)}{\bar{Y}_{i}^{2}(\breve{\theta})}\right] \boldsymbol{A}+\beta \boldsymbol{R}(\breve{\theta})\right]^{-1} \boldsymbol{A}^{\prime} D\left[\frac{1}{\bar{Y}_{i}(\breve{\theta})}\right] \boldsymbol{A} e^{j} .
$$

For moderate or small values of $\beta, \breve{\theta}$ is a slightly blurred version of $\theta$ (see (7)). Since the projection operation $\boldsymbol{A} \theta$ is a smoothing operator, the projections $\bar{Y}(\theta)$ and $\bar{Y}(\ddot{\theta})$ are approximately equal. Therefore, ${ }^{5}$ we simplify the above expression to

$$
l^{j}(\theta) \approx\left[\boldsymbol{A}^{\prime} D\left[u_{i}^{\mathrm{emis}}(\theta)\right] \boldsymbol{A}+\beta \boldsymbol{R}(\breve{\theta})\right]^{-1} \boldsymbol{A}^{\prime} D\left[u_{i}^{\mathrm{emis}}(\theta)\right] \boldsymbol{A} e^{j}
$$

where

$$
u_{i}^{\mathrm{emis}}(\theta)=\frac{1}{\bar{Y}_{i}(\theta)}
$$

is the reciprocal of the variance of $Y_{i}$ under the assumed Poisson model. For penalized-likelihood estimators in emission tomography, (18) is our final approximation to the local impulse response.

To summarize, we have derived a general local impulse response expression (14) for penalized-likelihood estimators, and specific expressions (18) for emission (and transmission [311) tomography.

\section{RESOlUtion PROPERTIES}

The local impulse response approximations for penalizedlikelihood image reconstruction in emission tomography (18) and transmission tomography [31] differ only by the definitions of the $u_{i}$ terms in the diagonal matrix. Thus, the local impulse response has the following generic form:

$$
l^{j}(\theta) \approx\left[\boldsymbol{A}^{\prime} \boldsymbol{D}_{\theta} \boldsymbol{A}+\beta \boldsymbol{R}(\breve{\theta})\right]^{-1} \boldsymbol{A}^{\prime} \boldsymbol{D}_{\theta} \boldsymbol{A} e^{j},
$$

${ }^{5}$ The diagonal terms in (18) and the preceding equation are sandwiched between the backprojection and projection operators $A^{\prime}$ and $A$, which smooth out most differences between $\bar{Y}(\theta)$ and $\bar{Y}(\breve{\theta})$. In a sense, the heavy-tailed $1 / r$ kernel that makes tomography ill-posed works to our advantage when making the above approximations. 
where $\boldsymbol{D}_{\theta}=D\left[u_{i}(\theta)\right]$ is an object-dependent diagonal matrix with $u_{i}(\theta)$ defined by (19) for emission tomography and, similarly [31], for transmission tomography.

Many penalty functions used in tomography can be written in the following form: ${ }^{6}$

$$
R(\theta)=\sum_{j=1}^{p} \frac{1}{2} \sum_{k \in \mathcal{N}_{j}} w_{j k} \psi\left(\theta_{j}-\theta_{k}\right)
$$

where $\mathcal{N}_{j}$ is a neighborhood of pixels near pixel $j, \psi$ is a symmetric convex function, and $w_{j k}=w_{k j}$. For a "first-order" neighborhood, one chooses $w_{j k}$ to equal one for horizontal and vertical neighboring pixels, and zero otherwise; for a "second-order" neighborhood, one also includes $w_{j k}=1 / \sqrt{2}$ for diagonal neighbors. With either of these standard choices for the $w_{j k}$ 's, we refer to $R(\theta)$ as a uniform penalty, since it is shift-invariant; i.e., translating the image yields an identical value of $R(\theta)$.

One of the simplest uniform penalties is the uniform quadratic penalty, which refers to the case where $\psi(x)=$ $x^{2} / 2$. In this case, the penalty has a quadratic form

$$
R(\theta)=\frac{1}{2} \theta^{\prime} \boldsymbol{R} \theta
$$

where $R$ is a $\theta$-independent $p \times p$ matrix defined by

$$
\boldsymbol{R}_{j k}= \begin{cases}\sum_{l \in \mathcal{N}_{j}} w_{j l}, & k=j \\ -w_{j k}, & k \neq j .\end{cases}
$$

In the quadratic case, the local impulse response simplifies to

$$
l^{j}(\theta) \approx\left[\boldsymbol{A}^{\prime} \boldsymbol{D}_{\theta} \boldsymbol{A}+\boldsymbol{\beta} \boldsymbol{R}\right]^{-1} \boldsymbol{A}^{\prime} \boldsymbol{D}_{\theta} \boldsymbol{A} e^{j}
$$

\section{A. Projection Dependence}

When $R(\theta)$ is a quadratic form so that $\boldsymbol{R}$ is independent of $\theta$, then, remarkably, the local impulse response approximation $l^{j}(\theta)$ given by (22) depends on the object $\theta$ only through its projections $\bar{Y}(\theta)$ (see (19)). Even if the object is unknown, its projections are approximately known through the noisy measurements $y$. Thus, even for real noisy measurements, we can predict the local impulse response simply by replacing $\bar{Y}(\theta)$ with $y$ in (18). This simple approach is effective primarily because the diagonal terms in (18) are sandwiched between the backprojection and projection operators $\boldsymbol{A}^{\prime}$ and $\boldsymbol{A}$, which greatly smooth out the noise in $y$, i.e.,

$$
\boldsymbol{A}^{\prime} D\left[\bar{Y}_{i}(\theta)\right]^{-1} \boldsymbol{A} \approx \boldsymbol{A}^{\prime} D\left[y_{i}\right]^{-1} \boldsymbol{A} .
$$

${ }^{6}$ If $\ddot{\psi}(x)>0$ for all $x$, then it is easily shown that the only vectors in the null space of the matrix $\nabla^{2} R(\theta)$ are of the form $v=1_{p} v_{1}$, where $1_{p}$ is the length- $p$ vector of ones. For any tomographic system that satisfies $D_{\theta} A 1_{p} \neq 0$ (i.e., the projection of a uniform image is nonzero), we can then conclude that $\boldsymbol{A}^{\prime} \boldsymbol{D}_{\theta} \boldsymbol{A}+\beta \boldsymbol{R}(\breve{\theta})$ is positive definite and therefore invertible, as required by (16).

\section{B. Nonuniformity}

One might expect that a uniform penalty such as (21) would induce uniform spatial resolution, just as space-invariant sieves do [2]. Using the preconditioned conjugate gradient [40], [22] or Gauss-Siedel [20], [21] algorithms, one can evaluate (20) or (22) and then display the local impulse response for several locations within the object. Upon doing this, one immediately finds that the local impulse response is very nonuniform, even for standard uniform quadratic penalties. (See Section VI.)

The next section elaborates on this property, but one can partially understand the source of the resolution nonuniformity by considering (22). If the measurement noise was homoscedastic with variance $\nu$, then $D$ would be simply a scaled identity matrix $D=\nu^{-1} I$, and from (22) the local impulse response would be

$$
\begin{aligned}
l^{j}(\theta) & =\left[\nu^{-1} \boldsymbol{A}^{\prime} \boldsymbol{A}+\beta \boldsymbol{R}\right]^{-1} \nu^{-1} \boldsymbol{A}^{\prime} \boldsymbol{A} e^{j} \\
& =\left[\boldsymbol{A}^{\prime} \boldsymbol{A}+\nu \beta \boldsymbol{R}\right]^{-1} \boldsymbol{A}^{\prime} \boldsymbol{A} e^{j} .
\end{aligned}
$$

In other words, noise with variance $\nu$ leads to an impulse response that corresponds to an "effective" smoothing parameter $\nu \beta$. Thus, the influence of the smoothing penalty is not invariant to changes in the noise variance, which perhaps explains in part why choosing $\beta$ is considered by many investigators to be a difficult process. The Poisson case is more complicated since the values of $\boldsymbol{D}_{\theta}$ vary along the diagonal. Since a given pixel is primarily affected by the detectors whose rays intersect it, each pixel sees a different "effective variance" and hence a different effective smoothing parameter.

This resolution nonuniformity can also be explained from a Bayesian perspective. The Fisher information $A^{\prime} D_{\theta} A$ is a measure of the certainty in the data. For pixels where this data certainty is smaller (due to higher noise variance in the rays that intersect that pixel), the posterior estimate will give more weight to the prior, which (being a smoothness prior) will cause more smoothing. In emission tomography, pixels with higher activity yield rays with higher counts and hence higher absolute variance or lower certainty. Paradoxically, penalizedlikelihood methods using the standard uniform penalty thus have lower spatial resolution in high-count regions. This property is certainly undesirable, and may explain in part why many authors have characterized the uniform quadratic penalty as causing "oversmoothing," since the most prominent image features are generally smoothed the most!

\section{Choosing $\beta$ for One Pixel}

Since (22) allows one to predict the local impulse response (and hence the spatial resolution) at any pixel $j$ as a function of $\beta$, one could use (22) to choose a value for $\beta$ that induces a desired resolution at some pixel $j$ of interest in the image. However, the induced resolutions at other points in the image would still be different, which motivates the modified penalty developed in the next section.

\section{RESOLUTION UNIFORMITY}

This section analyzes the problem of resolution nonuniformity more closely. This analysis leads to a natural modified 
penalty function that induces more uniform resolution. For simplicity, we focus on emission tomography; parallel arguments apply to transmission tomography.

\section{A. Emission Tomography}

In emission tomography, the Fisher information matrix $\boldsymbol{A}^{\prime} \boldsymbol{D}_{\theta} \boldsymbol{A}$ is an operator that, due to the lexicographic ordering of pixels, one can treat as a mapping from image space to image space. The operator $\boldsymbol{A}^{\prime} \boldsymbol{D}_{\theta} \boldsymbol{A}$ is shift variant for emission tomography, which is the crux of the problem of resolution nonuniformity. The previous section noted that the nonuniform diagonal of the $D_{\theta}$ term is partially responsible for the nonuniform local impulse response. But even without that term, the spatial resolution would still be nonuniform because typically even $\boldsymbol{A}^{\prime} \boldsymbol{A}$ is a shift-variant operator in PET and SPECT. However, one often models the system matrix $\boldsymbol{A}$ as a product of three factors: $a_{i j}=c_{i} g_{i j} s_{j}$, such that $\boldsymbol{G}^{\prime} \boldsymbol{G}$ is approximately shift invariant, where $\boldsymbol{G}=\left\{g_{i j}\right\}$ represents the object-independent ${ }^{7}$ geometric portion of the tomographic system response. The $c_{i}$ 's represent ray-dependent factors that change between studies, including detector efficiency factors, dead time, radioisotope decay, and (in PET) attenuation factors. The $s_{j}$ 's represent pixel-dependent factors such as spatial variations in sensitivity, and (in SPECT) "first-order" attenuation correction factors (cf the image-space Chang method [41] for SPECT attenuation correction). For our PET work thus far, we have simply used $s_{j}=1$. In matrix notation

$$
\boldsymbol{A}=D\left[c_{i}\right] \boldsymbol{G} D\left[s_{j}\right] .
$$

This factorization is not unique. If one desires resolution uniformity, then the analysis that follows suggests that one should strive to choose $\left\{c_{i}\right\}$ and $\left\{s_{j}\right\}$ so that $\boldsymbol{G}^{\prime} \boldsymbol{G}$ is "as shiftinvariant as possible" (cf (38) below). (See [42] for additional analyses of shift-invariant and shift-variant imaging systems.)

Substituting (25) into (18) and simplifying, we obtain

$$
\begin{gathered}
l^{j}(\theta) \approx\left[D\left[s_{j}\right] \boldsymbol{G}^{\prime} D\left[q_{i}(\theta)\right] \boldsymbol{G} D\left[s_{j}\right]+\beta \boldsymbol{R}(\breve{\theta})\right]^{-1} \\
\cdot D\left[s_{j}\right] \boldsymbol{G}^{\prime} D\left[q_{i}(\theta)\right] \boldsymbol{G} D\left[s_{j}\right] e^{j}
\end{gathered}
$$

where

$$
q_{i}(\theta)=c_{i}^{2} / \bar{Y}_{i}(\theta)
$$

In PET, these $q_{i}$ 's are very nonuniform due to attenuation correction factors that range from 1.0 to 100 , detector efficiencies that vary over an order of magnitude in block crystal systems, and the intrinsic count variations of Poisson measurements.

The Fisher information matrix for estimating $\theta$ is

$$
\boldsymbol{F}(\theta)=\boldsymbol{A}^{\prime} D\left[u_{i}(\theta)\right] \boldsymbol{A}=D\left[s_{j}\right] \boldsymbol{G}^{\prime} D\left[q_{i}(\theta)\right] \boldsymbol{G} D\left[s_{j}\right]
$$

As a consequence of the nonuniformity of the $q_{i}$ 's, the diagonal of $\boldsymbol{F}(\theta)$ is also nonuniform, which contributes greatly to the shift variance of the $\boldsymbol{F}(\theta)$ operator in PET.

${ }^{7}$ In SPECT, $\boldsymbol{G}$ will only be approximately object independent due to attenuation.
Understanding the structure of $\boldsymbol{F}(\theta)$ is the key to correcting the resolution nonuniformity. From (28), the diagonal elements of $\boldsymbol{F}(\theta)$ can be written

$$
\boldsymbol{F}_{j j}(\theta)=s_{j}^{2} \sum_{i} g_{i j}^{2} q_{i}(\theta)=\kappa_{j}^{2}(\theta) \sum_{i} g_{i j}^{2}, \quad j=1, \cdots, p
$$

where we define

$$
\kappa_{j}(\theta)=s_{j} \sqrt{\sum_{i} g_{i j}^{2} q_{i}(\theta) / \sum_{i} g_{i j}^{2}} .
$$

Due to the $1 / r$ response of tomographs, $\boldsymbol{F}(\theta)$ is fairly concentrated about its diagonal, so (29) suggests the approximation

$$
\boldsymbol{F}(\theta)=D\left[s_{j}\right] \boldsymbol{G}^{\prime} D\left[q_{i}(\theta)\right] \boldsymbol{G} D\left[s_{j}\right] \approx \Lambda_{\theta} \boldsymbol{G}^{\prime} \boldsymbol{G} \Lambda_{\theta},
$$

where

$$
\Lambda_{\theta}=D\left[\kappa_{j}(\theta)\right]
$$

is a $p \times p$ diagonal matrix. From (29), one sees that approximation (31) is exact along the diagonal of $\boldsymbol{F}(\theta)$, and would be exact on the off-diagonal elements if the $q_{i}$ 's were equal. The approximation (31) turns out to be reasonably accurate even for very nonuniform $q_{i}$ 's because the $\kappa_{j}$ 's vary slowly as a function of $j$ due to the smoothing implicit in (30). This approximation also reflects the fact that the local impulse response of pixel $j$ depends primarily on the $q_{i}$ 's that correspond to rays that intersect pixel $j$.

To visualize (31), Fig. 2 shows the various matrices for a toy PET problem ${ }^{8}$ (with $s_{j}=1$ ). The nearly Toeplitz-blockToeplitz structure of $G^{\prime} G$ is apparent.

Substituting (31) into (26) and rearranging yields the following approximation to the local impulse response:

$$
\begin{aligned}
l^{j}(\theta) & \approx\left[\Lambda_{\theta} \boldsymbol{G}^{\prime} \boldsymbol{G} \Lambda_{\theta}+\beta \boldsymbol{R}(\breve{\theta})\right]^{-1} \Lambda_{\theta} \boldsymbol{G}^{\prime} \boldsymbol{G} \Lambda_{\theta} e^{j} \\
& =\Lambda_{\theta}^{-1}\left[\boldsymbol{G}^{\prime} \boldsymbol{G}+\beta \Lambda_{\theta}^{-1} \boldsymbol{R}(\breve{\theta}) \Lambda_{\theta}^{-1}\right]^{-1} \boldsymbol{G}^{\prime} \boldsymbol{G} \Lambda_{\theta} e^{j} \\
& =\kappa_{j}(\theta) \Lambda_{\theta}^{-1}\left[\boldsymbol{G}^{\prime} \boldsymbol{G}+\beta \Lambda_{\theta}^{-1} \boldsymbol{R}(\breve{\theta}) \Lambda_{\theta}^{-1}\right]^{-1} \boldsymbol{G}^{\prime} \boldsymbol{G} e^{j}
\end{aligned}
$$

because $\Lambda_{\theta} e^{j}=\kappa_{j}(\theta) e^{j}$.

What does $\Lambda_{\theta}$ represent statistically? From (30), we see that $\kappa_{j}(\theta)$ is a normalized backprojection of $\left\{q_{i}\right\}$, where $q_{i}$ is the inverse of the variance of the $i$ th corrected measurement $y_{i} / c_{i}$. Thus, $\kappa_{j}(\theta)$ represents an aggregate certainty of the measurement rays that intersect the $j$ th pixel. Since the local impulse response $l^{j}$ is typically concentrated about pixel $j$, a somewhat cruder but nevertheless very useful approximation that follows from (33) is

$$
l^{j}(\theta) \approx\left[\boldsymbol{G}^{\prime} \boldsymbol{G}+\beta / \kappa_{j}^{2}(\theta) \boldsymbol{R}(\breve{\theta})\right]^{-1} \boldsymbol{G}^{\prime} \boldsymbol{G} e^{j}
$$

(cf. (24)). The accuracy of this approximation improves as $\beta$ decreases (and, hence, $l^{j}$ approaches the impulse $e^{j}$ ). This expression again illustrates the property that the effective amount of smoothing $\beta / \kappa_{j}^{2}(\theta)$ increases with decreasing measurement certainty $\kappa_{j}(\theta)$.

\footnotetext{
${ }^{8}$ The object was a $6 \times 2$ uniform rectangle in an $8 \times 6$ image. We used $c_{i}=1$, so the only nonuniformity in the $q_{i}$ 's was due to the $1 / \bar{Y}_{i}(\theta)$ contribution of Poisson noise.
} 

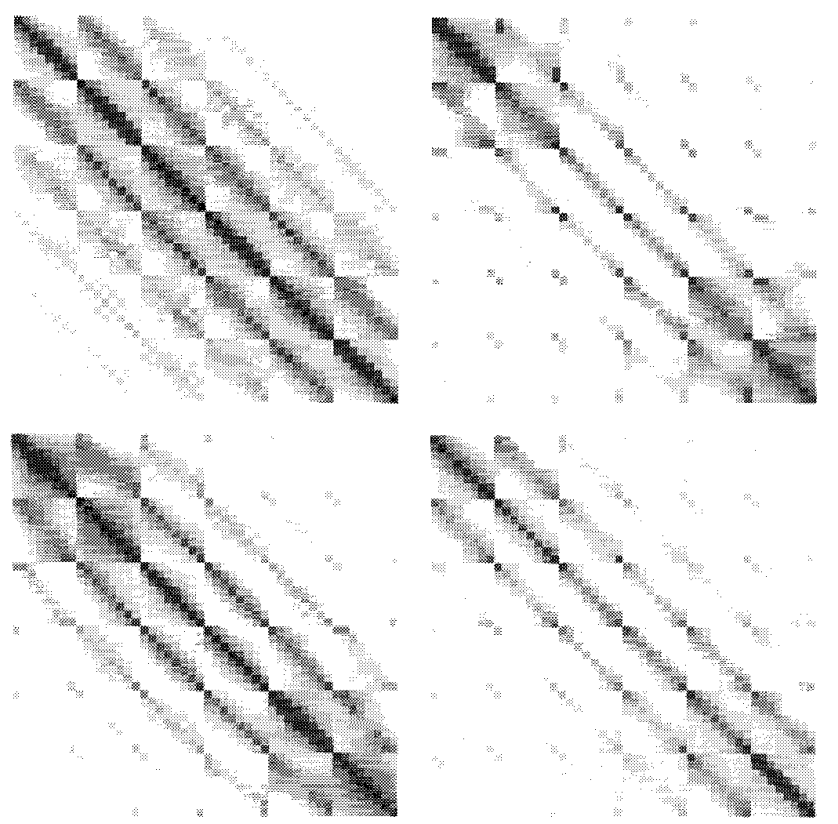

Fig. 2. Illustration of the approximation (31). Upper left: the matrix $G^{\prime} G$, which is approximately Toeplitz-block-Toeplitz. Upper right: the Fisher information $\boldsymbol{F}=\boldsymbol{G}^{\prime} D\left[q_{i}(\theta)\right] \boldsymbol{G}$ including Poisson noise covariance. The nonuniform diagonal is caused by the nonuniform Poisson noise variance. Lower right: the approximation $\Lambda G^{\prime} G \Lambda$; note the agreement with the upper right matrix, i.e., $\boldsymbol{F} \approx \Delta \boldsymbol{G}^{\prime} \boldsymbol{G} \Lambda$. Lower left: $\Lambda^{-1} \boldsymbol{G}^{\prime} D\left[q_{i}(\theta)\right] G \Lambda^{-1}$; note that this matrix is a reasonable approximation to $G^{\prime} G$.

Approximation (34) illuminates the paradoxical oversmoothing of high-count regions with the uniform penalty. If pixel $j$ is transected by rays with high counts, then from (27) and (30) we see that $q_{i}$ and, hence, $\kappa_{j}(\theta)$ will be small, so the effective smoothing parameter $\beta / \kappa_{j}^{2}(\theta)$ above will be large, causing lower resolution. As $\theta_{j}$ increases, the rays that intersect it will also increase, so the local resolution decreases. ${ }^{9}$

\section{B. A Modified Penalty}

The form of (33) suggests several possible methods for modifying the penalty function to improve resolution uniformity. We focus on one approach that is easily implemented. Let $R^{\star}(\theta)$ denote a "target" penalty function of the form (21) (presumably shift invariant) whose properties would be suitable if we had $\boldsymbol{D}_{\theta}=I$. Suppose we have estimates $\left\{\hat{\kappa}_{j}\right\}$ of $\left\{\kappa_{j}(\theta)\right\}$, and consider the modified penalty

$$
R(\theta)=\frac{1}{2} \sum_{j} \sum_{k \in \mathcal{N}_{j}} w_{j k} \hat{\kappa}_{j} \hat{\kappa}_{k} \psi\left(\theta_{j}-\theta_{k}\right)
$$

If $\boldsymbol{R}(\theta)=\nabla^{2} R(\theta)$ denotes the Hessian of this modified penalty, ${ }^{10}$ then one can show that

$$
\boldsymbol{R}_{j k}(\theta)= \begin{cases}\sum_{l \neq j} w_{j l} \hat{\kappa}_{j} \hat{\kappa}_{l} \ddot{\psi}\left(\theta_{j}-\theta_{k}\right), & j=k \\ -w_{j k} \hat{\kappa}_{j} \hat{\kappa}_{k} \ddot{\psi}\left(\theta_{j}-\theta_{k}\right), & j \neq k\end{cases}
$$

\footnotetext{
${ }^{9}$ However, note that even uniform objects (e.g. $\theta=[1 \cdots 1]$ ) lead to nonuniform resolution (i.e. to shift-variant local impulse response), since $\bar{Y}(\theta)$ will be a nonuniform vector due to the different lengths of the line integrals through the object.

${ }^{10}$ One can easily verify that this Hessian is nonnegative definite if $\ddot{\psi}>0$.
}

so that if $D\left[\hat{\kappa}_{j}\right] \approx \Lambda_{\theta}$ and we let $\boldsymbol{R}^{\star}(\theta)=\nabla^{2} R^{\star}(\theta)$, then

$$
\boldsymbol{R}(\theta) \approx \Lambda_{\theta} \boldsymbol{R}^{\star}(\theta) \Lambda_{\theta} .
$$

This approximation relies on the fact that neighboring pixels have very similar certainties, i.e., $\kappa_{k}(\theta) \approx \kappa_{j}(\theta)$ for $k \in$ $\mathcal{N}_{j}$, which again follows from the smoothing effect of (30). Substituting (36) into the expression for the local impulse response (33) yields the new approximation

$$
l^{j}(\theta) \approx \kappa_{j}(\theta) \Lambda_{\theta}^{-1}\left[\boldsymbol{G}^{\prime} \boldsymbol{G}+\beta \boldsymbol{R}^{\star}(\breve{\theta})\right]^{-1} \boldsymbol{G}^{\prime} \boldsymbol{G} e^{j} .
$$

If the geometric response $\boldsymbol{G}$ is nearly space invariant, then to within our approximation accuracy, (37) corresponds to nearly uniform resolution except for the following features.

- Unlike the uniform quadratic target penalty, for which $\boldsymbol{R}^{\star}$ is constant along its diagonal, nonquadratic penalties lead to object-dependent Hessians $\boldsymbol{R}^{\star}(\breve{\theta})$. However, users of nonquadratic penalties presumably desire certain nonuniformities, i.e. more smoothing in flat regions and less smoothing near edges. Our modified penalty (35) preserves this important characteristic of nonquadratic penalties. Our modification only corrects for the resolution nonuniformities that are induced by the interaction between the nonuniform statistics and the penalty function. Essentially we are correcting for the $\Lambda_{\theta}^{-1} R \Lambda_{\theta}^{-1}$ term in (33).

- Since $\kappa_{j}(\theta) / \kappa_{k}(\theta) \approx 1$ for $k \in \mathcal{N}_{j}$, the term $\kappa_{j}(\theta) \Lambda_{\theta}^{-1}$ in (37) effectively acts as an identity matrix for pixels near $j$, so for local impulse responses that are fairly narrow we can disregard the $\kappa_{j}(\theta) \Lambda_{\theta}^{-1}$ term, leading to the approximation

$$
l^{j}(\theta) \approx\left[\boldsymbol{G}^{\prime} \boldsymbol{G}+\beta \boldsymbol{R}^{\star}(\breve{\theta})\right]^{-1} \boldsymbol{G}^{\prime} \boldsymbol{G} e^{j} .
$$

By "narrow" we mean relative to the scale of the spatial fluctuations in $\kappa_{j}(\theta)$. However, in regions where the certainty $\kappa_{j}(\theta)$ is more rapidly varying as a function of spatial position (such as near the edge of an object), the presence of the term $\kappa_{j}(\theta) \Lambda_{\theta}^{-1}$ indicates that there will be some asymmetry in the local impulse response. As illustrated in Section VI, such asymmetry can occur with or without our modifications to the penalty. Further work is needed to correct these asymmetries.

\section{Practical Implementation}

In practice, the term $\kappa_{j}(\theta)$ is unknown, since it depends on the noiseless measurement mean $\bar{Y}(\theta)$. Fortunately, we can manipulate the noisy data to provide a reasonable estimate $\hat{\kappa}_{j}$ of $\kappa_{j}(\theta)$.

We first compute from the measurements an estimate $\hat{q}_{i}$ of the term $q_{i}(\theta)$ defined by $(27)$ :

$$
\hat{q}_{i}=\frac{c_{i}^{2}}{\max \left\{y_{i}, 10\right\}} .
$$

The " 10 " factor ensures that the denominator is not too close to zero, and hopefully provides a little robustness to model mismatch by giving no rays an inordinate weighting. We then replace the $q_{i}(\theta)$ term in (30) with $\hat{q}_{i}$ to precompute $\hat{\kappa}_{j}$, which we then use in (35). Thus, implementing the modified 
penalty (35) simply requires one extra backprojection. (To save a little computation, one could probably replace (30) with an approximate backprojector.) The cost of multiplying by $\hat{\kappa}_{j} \hat{\kappa}_{k}$ in (35) is negligible compared to the forward projections required by iterative reconstruction algorithms.

Since the $\hat{\kappa}_{j}$ 's depend on the data, our modified penalty (35) is data-dependent! Bayesian-minded readers may find the idea of a data-dependent "prior" to be somewhat disconcerting. We make absolutely no pretense that this approach has any Bayesian interpretation. The purpose of the penalty is solely to control noise, and the purpose of our modification to the penalty is solely to control the resolution properties. As an alternative to (39), one could periodically update the $\hat{\kappa}_{j}$ 's by substituting one's current estimate of $\hat{\theta}$ into (30) within an iterative algorithm. But the extra effort is unlikely to change the final estimate very much, since, as noted earlier, small changes in the $q_{i}$ 's have minor effects on the estimate due to the "sandwich" effect described in footnote 5 and by (23).

Since (35) and (39) define the modified penalty $R(\theta)$ to be a function that depends on $y$, the matrix $\nabla^{11} R$ is no longer exactly zero, so strictly speaking the steps between (14) and (16) need modification. However, because of the effective smoothing in the definition (30), the partial derivatives with respect to $y$ of the modified penalty are very small, so we ignore this second-order effect.

\section{Choosing $\beta$}

For a quadratic target penalty $R^{\star}(\theta)$, the local impulse response (38) induced by our modified penalty (35) is independent of the object $\theta$. Thus, the process of choosing the smoothing parameter $\beta$ is significantly simplified by the following approach. Let $j$ be a pixel in the center of the image, for example. For a given system geometric response $\boldsymbol{G}$, precompute the local impulse response (38) for a range of values of $\beta$. For each $\beta$, tabulate some measure of resolution, such as the FWHM of $l^{j}$. Then, when presented with a new data set to be reconstructed at some user-specified resolution, simply interpolate the table to determine the appropriate value for $\beta$. Finally, reconstruct the object using the modified quadratic penalty. Section VI presents results that demonstrate the effectiveness of this approach. Analytical results in [31] further simplify the process of building this table for certain tomographs.

Many (but not all) nonquadratic penalties are locally quadratic near zero, and it is this quadratic portion of the penalty that is active within relatively flat regions in the image. For such penalties, one could use the table approach described above to specify the desired "resolution" in the flat parts of the image, and then adjust any remaining penalty parameters to control the influence of edges etc. For penalties that are not even locally quadratic, such as the generalized Gaussian-Markov random field prior [32], further investigation is needed.

\section{EXAMPLES}

This section demonstrates the improved resolution uniformity induced by the modified penalty (35) within a penalized-

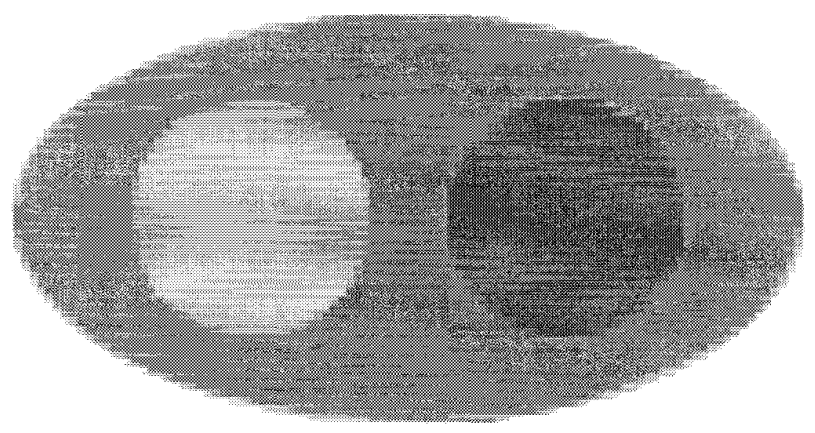

Fig. 3. Digital phantom used to examine spatial resolution properties.

likelihood image reconstruction method for PET emission measurements. For $\theta$, we used the $128 \times 64$ emission image shown in Fig. 3, which has relative emission intensities of 1,2 , and 3 in the cold disk (left), background ellipse, and hot disk (right), respectively. We included the effects of nonuniform attenuation in the $c_{i}$ 's by using an attenuation map qualitatively similar to Fig. 3, but with attenuation coefficients $0.003,0.0096$, and $0.013 / \mathrm{mm}$ for the cold disk, background ellipse, and hot disk, respectively. The pixel size was $3 \mathrm{~mm}$. Rather than being anthropomorphic, this phantom was designed to demonstrate that the modified penalty induces nearly uniform spatial resolution even for problems where the standard penalty yields highly nonuniform spatial resolution.

We simulated a PET emission scan with 128 radial bins and 110 angles uniformly spaced over $180^{\circ}$. The $g_{i j}$ factors corresponded to 6-mm-wide strip integrals with 3-mm centerto-center spacing. We set $r_{i}=0.1(1 / N) \Sigma_{i^{\prime}} \Sigma_{j} a_{i^{\prime} j} \theta_{j}$, which corresponds to $10 \%$ random coincidences.

\section{A. Resolution Uniformity}

We computed local impulse response functions $l^{j}(\theta)$ for three pixels $j$, corresponding to the center of the cold disk, the center of the image, and the center of the hot disk. We used the recipe following (9) with $\delta=0.01$ to evaluate $l^{j}(\theta)$, for both the standard penalty (21) and the modified penalty (35) with $\psi(x)=x^{2} / 2$. For both penalties, we used a firstorder neighborhood. We used this recipe rather than any of the approximations that followed it (such as (18)) to provide a more convincing demonstration; for routine work we usually just use (26). (The results of (26) are not shown in Fig. 4 since they were indistinguishable from the curves shown, which supports the accuracy of the approximations leading to (26).) We maximized the objective function (15) to compute $\hat{\theta}$ in (5) using 20 iterations of the PML-SAGE-3 algorithm [18].

Fig. 4 displays horizontal and vertical profiles through the local impulse responses for the estimators corresponding to the two penalty functions. The circles in Fig. 4 are for the unbiased estimator (6) for $M=2000$ realizations. The standard penalty has highly nonuniform spatial resolution, whereas the modified penalty yields nearly uniform spatial resolution. These results are typical.

\section{B. Asymmetry}

In part because of the large eccentricity of the ellipse in Fig. 3, the local impulse responses of both penalties are 

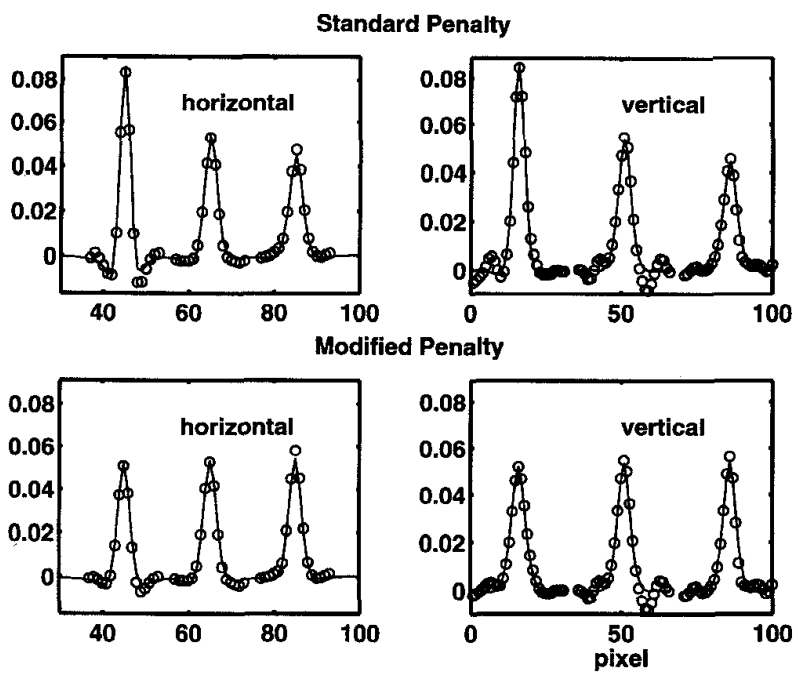

Fig. 4. Horizontal and vertical profiles (concatenated left to right) through three local impulse response functions for a penalized-likelihood estimate of the image shown in Fig. 3. The standard quadratic penalty yields highly nonuniform resolution (upper profiles), whereas the proposed modified penalty leads to nearly uniform spatial resolution (lower profiles). Note that for the standard penalty the resolution is poorest in the high-count (rightmost) disk.
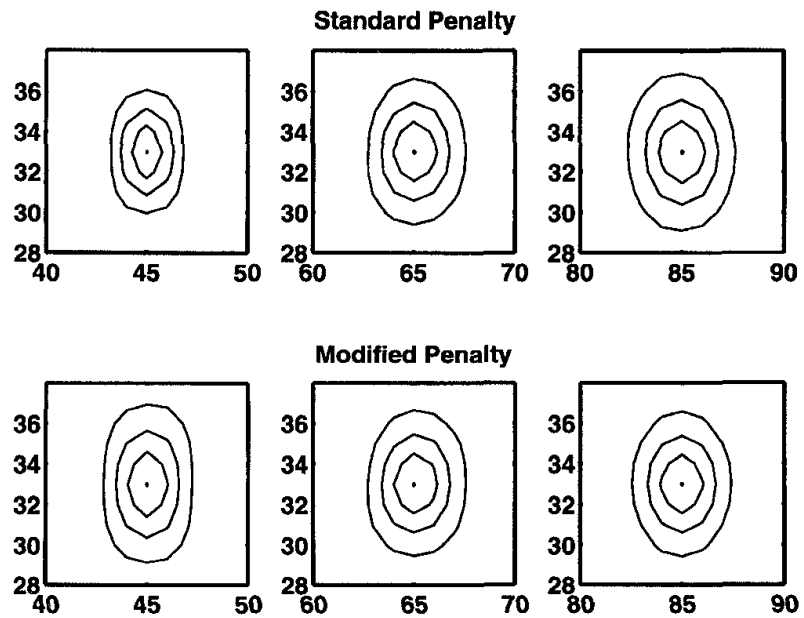

Fig. 5. Contours of the local impulse response functions at 25, 50, 75, and $99 \%$ of each peak. Left: center of cold disk; middle: center of image; right: center of hot disk.

asymmetric. Fig. 5 displays contours at levels 25, 50, 75, and $99 \%$ of the peak value for each PSF, computed using the contour function of Matlab. We hope to extend the analysis in this paper to develop suitable modifications to the penalty that will reduce this asymmetry. (The corresponding contours for FBP were virtually circular.)

\section{Choosing $\beta$}

We now describe how we selected $\beta$ for this simulation, which illustrates the effectiveness of the table-based approach described in Section V-D. First, we decided for illustration purposes to use a FWHM of 4.0 pixels. Using the analytical results detailed in [31] for the system geometry described above, the value $\beta=2^{-4.44}$ is required for the modified penalty. ${ }^{11}$ Did this choice of $\beta$ actually give the desired 4.0 pixels FWHM resolution? Since Fig. 5 shows that the local impulse response is asymmetric, clearly the resolution is not exactly 4.0 pixels FWHM isotropically. In particular, for the same three pixels considered above, the horizontal resolutions were $3.10,3.38$, and 3.34 pixels FWHM, whereas the vertical resolutions were 5.28, 4.83, and 4.76 pixels FWHM. However, the averages of the horizontal and vertical resolutions were $4.19,4.10$, and 4.05 pixels FWHM, all of which are within $5 \%$ of the target resolution of 4.0 pixels FWHM. Thus, although further work is needed to correct the asymmetry in such eccentric objects, the proposed method for selecting $\beta$ appears to yield local impulse responses whose average resolution is very close to the desired resolution. These results are typical in our experience.

\section{WHAT HAPPENS TO THE VARIANCE?}

It is well known that the global smoothing parameter $\beta$ controls an overall tradeoff between resolution and noise: larger $\beta$ 's lead to coarser resolution but less noise, and viceversa. The analysis in preceding sections shows that for the modified penalty to induce uniform spatial resolution, the "local" smoothing parameter must effectively be larger in some areas, and smaller in others. Thus, it is natural to expect that these changes in the local resolution will also influence the noise-but is the influence global or local? In other words, if the modified penalty increases the resolution (and hence the noise) at a given pixel, will that noise somehow propagate to distant pixels and lead to an overall worse resolution/noise tradeoff?

To address this question, we generated 100 realizations of Poisson distributed simulated PET measurements for the object shown in Fig. 3, and for the system properties described in Section VI. For each realization $y^{(1)}, \cdots, y^{(100)}$, we used 20 iterations of PML-SAGE-3 [18] to compute penalizedlikelihood estimates $\left\{\hat{\theta}\left(y^{(m)}\right)\right\}_{m=1}^{100}$ for several values of $\beta$ for both the standard and the modified quadratic penalties. For each value of $\beta$, we computed the empirical standard deviation of $\hat{\theta}_{j}$ for the pixels at the centers of the two disks in Fig. 3. (The results were similar for the pixel at the image center, so are not shown.)

\section{A. Just What You Expected}

Fig. 6 shows the tradeoff between resolution (measured by the average FWHM of the local impulse response) and noise (measured by the empirical standard deviation) as $\beta$ is varied. Fig. 6 also shows predicted standard deviations computing using the variance approximations described in [38]. (The good agreement between empirical and predicted results in Fig. 6 is further confirmation of the utility of the approximations in [38].)

In Fig. 6, the resolution/noise data points follow an essentially identical tradeoff curve for both the standard and the

\footnotetext{
${ }^{11}$ For the standard penalty, we used the above value of $\beta$ scaled down by $\hat{\kappa}_{j}^{2}$ for the single $j$ corresponding to the pixel at the center of the image, as suggested by (34) and described in Section IV-C. This choice matched the resolution at the image center for the two penalties, as illustrated in the center plots of Figs. 4 and 5.
} 


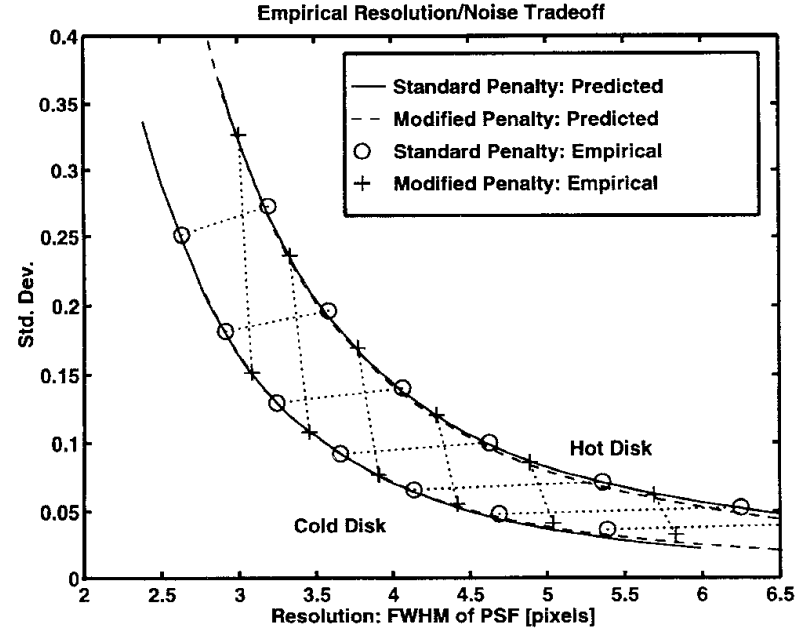

Fig. 6. Resolution/noise tradeoff for penalized-likelihood emission image reconstruction with standard and modified quadratic penalties. The two penalties induce virtually identical tradeoff curves. (The dotted lines connect points that correspond to the same $\beta$ value.)

modified penalty. This is true both for the analytically predicted tradeoff (the solid line and the dashed line overlap almost perfectly) as well as for the empirical results (the circle and the plus symbols lie on the same curve). These results suggest that the effects of the modified penalty are essentially local: A given pixel moves up or down its own resolution/noise tradeoff curve to the specified resolution, and then has a variance that is the same value as would be obtained if one were to use the standard penalty but globally adjust $\beta$ to enforce that specified resolution at the given pixel. This property probably hinges on the fact that the $\kappa_{j}$ factors are spatially smooth. If one were to artificially create a $\kappa_{j}$ map having discontinuities and then apply the modification (35), then it is plausible that the results would be less regular than indicated in Fig. 6. Readers who apply variations of (35) to induce some type of data-based nonuniform resolution will need to consider the resolution/noise tradeoff in more detail.

Fig. 7 shows central horizontal profiles through empirical standard deviation maps of the penalized likelihood estimates for both the modified and the standard quadratic penalties. Also shown is a calculated prediction of the variance, an approximation developed in [31]. As noted in footnote 11, the penalties were chosen to have matched resolution at the image center, and in Fig. 7 the estimator variance is also matched at the image center. Note, however, that whereas the variance for the standard penalty is fairly uniform (at least for this object), the variance for the modified penalty is nonuniform. (Of course as we have shown it is the other way around for the spatial resolution.) This nonuniformity is consistent with the results of Fig. 6 . The relative variance is more uniform for the modified penalty.

\section{B. Quadratic Penalties Are Useful}

Fig. 8 compares the resolution/noise tradeoff of penalized likelihood with that of images reconstructed by FBP with a

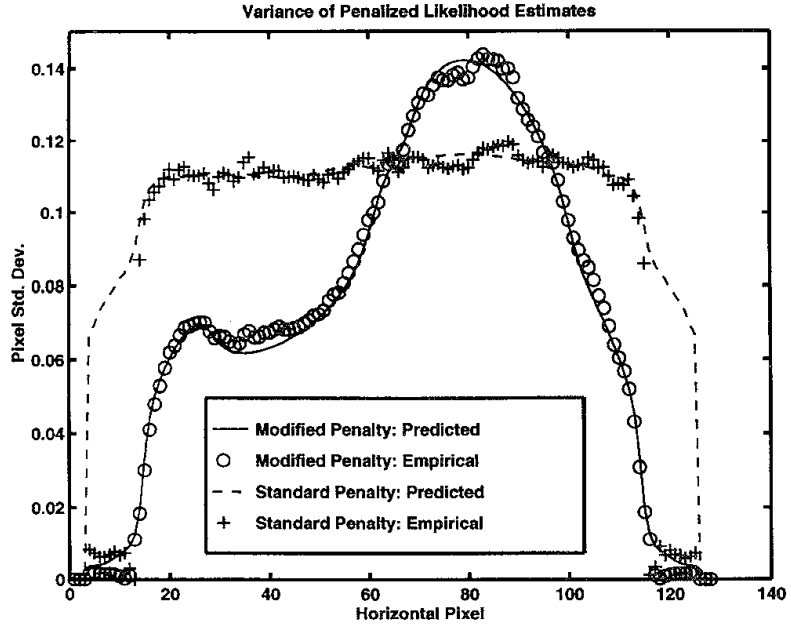

Fig. 7. Central horizontal profiles through empirical standard deviation maps for penalized likelihood emission estimates with the standard and modified penalties.

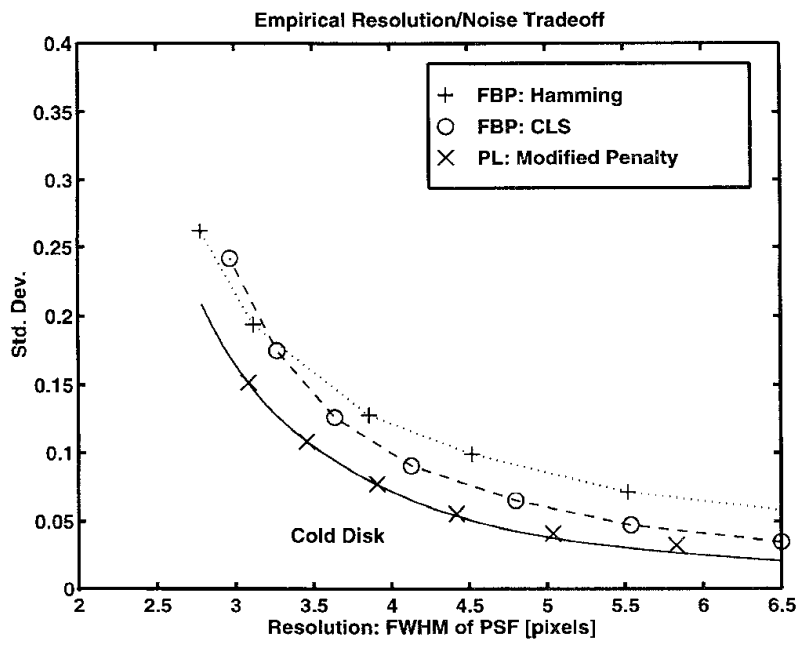

Fig. 8. Resolution/noise tradeoff of FBP with Hamming window and the constrained least-squares (CLS) window (40). At any given resolution, the variances of the penalized-likelihood estimates are smaller than those of FBP.

Hanning window and with the following constrained leastsquares (CLS) window developed in [31]:

$$
\frac{\operatorname{sinc}(2 u) / \operatorname{sinc}(u)}{\operatorname{sinc}^{2}(2 u)+\beta u^{3}}, \quad u \in\left[0, \frac{1}{2}\right],
$$

(where $u$ denotes spatial frequency: cycles per radial sample). This window induces a PSF indistinguishable from that of penalized-likelihood estimates with the first-order quadratic penalty [31]. As shown by Fig. 8, at any given resolution the empirical standard deviations for the FBP images are higher than for the penalized-likelihood estimates. This demonstrates that even using the oft-maligned quadratic penalty, penalizedlikelihood image reconstruction can outperform FBP in terms of the tradeoff between resolution and noise. Of course, non- 
quadratic prior models may give even better results for objects that are consistent with those models, but results such as Fig. 8 show that quadratic penalties provide a useful reduction in image noise over a large range of spatial resolutions.

\section{DISCUSSION}

We have analyzed the local impulse response of implicitly defined estimators (14) and of penalized-likelihood estimators for emission tomography (18) and transmission tomography [31]. The analysis and empirical results show that the local impulse response is asymmetric and has nonuniform resolution for Poisson distributed measurements. We proposed a modified regularization penalty (35) that improved the spatial resolution uniformity but not the asymmetry.

For the space-invariant tomographs considered here, the resolution nonuniformity arises from the nonuniform diagonal of the Fisher information matrix, which in turn is a consequence of the nonuniform variance of Poisson noise. In principle, one could "avoid" this problem altogether by using an unweighted least-squares estimator. We have shown qualitatively in [21] that nonuniform weighting is essential to achieve the desirable noise properties of statistical methods. In [31], we provide additional analyses and quantitative results that demonstrate the importance of weighting. Therefore, we advocate retaining the nonuniform weighting that is natural for Poisson statistics, but modifying the penalty to compensate for the undesirable spatial resolution properties. Fortunately, this modification does not destroy the benefits of the weighting, as shown in [31] and in Fig. 8, apparently because the nonuniform weighting is applied in sinogram space, whereas the penalty acts on the image space. It is an open question as to whether the modified penalty would be effective for problems such as restoration of quantum-limited image measurements, where both the unknown parameters and the data are images.

Some colleagues have argued that nonuniform resolution is desirable and expected. This opinion is presumably based on the idea that statistical methods can make better use of the measurement information and, thus, provide higher resolution in high-count regions. Ironically, our analysis shows that the effect of uniform penalties is just the opposite: More smoothing occurs in high-count regions. Although we have emphasized methods for achieving resolution uniformity, one could apply our analysis to develop alternative modified penalties that yield higher resolution in high-count regions according to some user-specified criterion. Since we now see that the statistics of the data themselves do not automatically provide a natural resolution-noise tradeoff in penalized-likelihood estimators (contrary to what may have been a widely held misconception), any such user-specified criteria will probably be considered somewhat arbitrary.

We have shown the somewhat remarkable result that the local impulse response induced by quadratic penalties depends on the object only through its projections. Thus, one does not need to know the object to predict the reconstructed resolution, since the noisy measurements serve as an adequate approximation to the object's projections. In contrast, the local impulse response for nonquadratic penalties depends explicitly on the (unknown) object (cf. (20)) through the Hessian of the penalty. Being able to predict and control the resolution properties induced by such penalty functions remains an important challenge.

For nonquadratic edge-preserving potential functions $\psi$, the nonuniform diagonal in (20) may induce additional types of nonuniformities beyond the resolution effects reported here. Specifically, we conjecture that the "propensity to retain edges" (as opposed to smoothing them out) will be space variant, again due to coupling between the Hessian of the $\log$-likelihood and the Hessian of the penalty in (20). If so, then modified penalties such as (35) may be useful for restoring the (presumably desirable) space invariance of the effects of edge-preserving penalties. The importance of such modifications is more likely to appear in rigorous studies of the ensemble characteristics of edge-preserving methods, rather than in anecdotal examples.

This paper has emphasized space-invariant tomographs. Further investigation is needed for space-variant systems such as SPECT emission measurements and truncated data such as fanbeam transmission SPECT and three-dimensional cylindrical PET.

Software related to this paper can be obtained from http://www.eecs.umich.edu/ fessler.

\section{ACKNOWLEDGMENT}

J. Fessler gratefully acknowledges an enlightening discussion with $\mathrm{H}$. Barrett, who pointed out the nonuniform sensitivity problem in SPECT, and wishes to thank an insightful reviewer for suggesting (6).

\section{REFERENCES}

[1] E. Veklerov and J. Llacer, "Stopping rule for the MLE algorithm based on statistical hypothesis testing," IEEE Trans. Med. Imaging, vol. MI-6, no. 4, pp. 313-319, Dec. 1987.

[2] D. L. Snyder, M. I. Miller, L. J. Thomas, and D. G. Politte, "Noise and edge artifacts in maximum-likelihood reconstructions for emission tomography," IEEE Trans. Med. Imaging, vol. MI-6, no. 3, pp. 228-238, Sept. 1987.

[3] R. M. Lewitt, "Multidimensional digital image representations using generalized Kaiser-Bessel window functions," J. Opt. Soc. Amer. Ser. $A$, vol. 7 , no. 10 , pp. $1834-1846$, Oct. 1990 .

[4] K. Lange, "Convergence of EM image reconstruction algorithms with Gibbs smoothing," IEEE Trans. Med. Imaging, vol. 9, no. 4, pp. 439-446, Dec. 1990.

[5] R. Leahy and X. H. Yan, "Statistical models and methods for PET image reconstruction," in Proc. Stat. Comput. Sect. Amer. Stat. Assoc., 1991, pp. $1-10$.

[6] J. A. Fessler, N. H. Clinthorne, and W. L. Rogers, "Regularized emission image reconstruction using imperfect side information," IEEE Trans. Nucl. Sci., vol. 39, no. 5, pp. 1464-1471, Oct. 1992.

[7] J. A. Fessler and W. L. Rogers, "Uniform quadratic penalties cause nonuniform image resolution (and sometimes vice versa)," in Conf. Rec. IEEE Nucl. Sci. Symp. Med. Imaging Conf., 1994, vol. 4, pp. 1915-1919.

[8] S. J. Reeves, "Optimal space-varying regularization in iterative image restoration," IEEE Trans. Image Processing, vol. 3, no. 3, pp. 319-323, May 1994.

[9] J. Nunez and J. Llacer, "Variable resolution Bayesian image reconstruction," in Proc. IEEE Workshop on Nonlinear Signal and Image Processing, 1995.

[10] J. A. Stamos, W. L. Rogers, N. H. Clinthorne, and K. F. Koral, "Object-dependent performance comparison of two iterative reconstruction algorithms," IEEE Trans. Nucl. Sci., vol. 35, no. 1, pp. 611-614, Feb. 1988. 
[11] D. W. Wilson and B. M. W. Tsui, "Spatial resolution properties of FB and ML-EM reconstruction methods," in Conf. Rec. IEEE Nucl. Sci. Symp. Med. Imaging Conf., vol. 2, pp. 1189-1193, 1993.

[12] structed emission tomographic images," IEEE Trans. Nucl. Sci., vol. 40, no. 4, pp. 1198-1203, Aug. 1993 .

[13] H. H. Barrett, D. W. Wilson, and B. M. W. Tsui, "Noise properties of the EM algorithm: I. Theory," Phys. Med. Biol., vol. 39, pp. 833-846, 1994

[14] D. W. Wilson, B. M. W. Tsui, and H. H. Barrett, "Noise properties of the EM algorithm: II. Monte Carlo simulations," Phys. Med. Biol, , vol. 39, pp. 847-872, 1994.

[15] J. S. Liow and S. C. Strother, "The convergence of object dependent resolution in maximum likelihood based tomographic image reconstruction," Phys. Med. Biol., vol. 38, no. 1, pp. 55-70, Jan. 1993.

[16] C. Bouman and K. Sauer, "A unified approach to statistical tomography using coordinate descent optimization," IEEE Trans. Image Processing, vol. 5, no. 3, pp. 480-492, Mar. 1996.

[17] E. U. Mumcuoglu, R. Leahy, S. R. Cherry, and Z. Zhou, "Fast gradientbased methods for Bayesian reconstruction of transmission and emission PET images," IEEE Trans. Med. Imaging, vol. 13, no. 3, pp. 687-701, Dec. 1994.

[18] J. A. Fessler and A. O. Hero, "Penalized maximum-likelihood image reconstruction using space-alternating generalized EM algorithms," IEEE Trans. Image Processing, vol. 4, no. 10, pp. 1417-29, Oct. 1995.

[19] J. A. Fessler, E. P. Ficaro, N. H. Clinthorne, and K. Lange, "Fast parallelizable algorithms for transmission image reconstruction," in Conf. Rec. IEEE Nucl. Sci. Symp. Med. Imaging Conf., 1995, vol. 3, pp. $1346-1350$

[20] K. Sauer and C. Bouman, "A local update strategy for iterative reconstruction from projections," IEEE Trans. Signal Processing, vol. 41, no. 2, pp. 534-548, Feb. 1993.

[21] J. A. Fessler, "Penalized weighted least-squares image reconstruction for positron emission tomography," IEEE Trans. Med. Imaging, vol. 13 no. 2, pp. 290-300, June 1994.

[22] S. D. Booth and J. A. Fessler, "Combined diagonal/Fourier preconditioning methods for image reconstruction in emission tomography" in Proc. IEEE Int. Conf. Image Processing, 1995, vol. 2, pp. 441-444.

[23] G. T. Gullberg and T. F. Budinger, "The use of filtering methods to compensate for constant attenuation in single-photon emission computed tomography," IEEE Trans. Biomed. Eng., vol. 28, no. 2, pp. 142-157, Feb. 1981 .

[24] Y. Pawitan and F. O'Sullivan, "Data-dependent bandwidth selection for emission computed tomography reconstruction," IEEE Trans. Med. Imaging, vol. 12, no. 2, pp. 167-172, June 1993.

[25] N. P. Galatsanos and A. K. Katsaggelos, "Methods for choosing the regularization parameter and estimating the noise variance in image restoration and their relation," IEEE Trans. Image Processing, vol. 1 no. 3, pp. 322-336, July 1992.

[26] J. W. Hilgers and W. R. Reynolds, "Instabilities in the optimal regularization parameter relating to image recovery problems," J. Opt. Soc Amer. Ser. A, vol. 9, no. 8, pp. 1273-1279, Aug. 1992.

[27] L. Kaufman and A. Neumaier, "Image reconstruction through regularization by envelope guided conjugate gradients," Bell Labs. Comp. Sci. Memo 11274-94-14, 1994.

[28] T. J. Hebert and R. Leahy, "Statistic-based MAP image reconstruction from Poisson data using Gibbs priors," IEEE Trans. Signal Processing, vol. 40, no. 9, pp. 2290-2303, Sept. 1992.

[29] T. R. Miller and J. W. Wallis, "Clinically important characteristics of maximum-likelihood reconstruction," J. Nucl. Med., vol. 33, no. 9, pp. 1678-1684, Sept. 1992.

[30] E. J. Hoffman, S. C. Huang, D. Plummer, and M. E. Phelps, "Quantitation in positron emission tomography: 6 effect of nonuniform resolution," J. Comput. Assisted Tomog., vol. 6, no. 5, pp. 987-999, Oct. 1982.

[31] J. A. Fessler, "Resolution properties of regularized image reconstruction methods," Tech. Rep. 297, Commun. and Signal Processing Lab., Dept. of Elect. Eng. Comput. Sci, Univ. Mich., Ann Arbor, Aug. 1995.

[32] C. Bouman and K. Sauer, "A generalized Gaussian image model for edge-preserving MAP estimation," IEEE Trans. Image Processing, vol. 2, no. 3, pp. 296-310, July 1993.

[33] A. K. Jain, Fundamentals of Digital Image Processing. Englewood Cliffs, NJ: Prentice-Hall, 1989.
[34] A. O. Hero, J. A. Fessler, and M. Usman, "Exploring estimator biasvariance tradeoffs using the uniform CR bound," IEEE Trans. Signal Processing, vol. 44, no. 8, pp. 2026-2041, Aug. 1996.

[35] M. Usman, A. O. Hero, and J. A. Fessler, "Uniform CR bound: implementation issues and applications," in Conf. Rec. IEEE Nucl. Sci. Symp. Med. Image Conf., vol. 3, pp. 1443-1447, 1994.

[36] , "Bias-variance tradeoffs analysis using uniform $\mathrm{CR}$ bound for image reconstruction," in Proc. IEEE Int. Conf. Image Processing, vol. 2, pp. 835-839, 1994.

[37] R. E. Carson, Y. Yan, B. Chodkowski, T. K. Yap, and M. E. DaubeWitherspoon, "Precision and accuracy of regional radioactivity quantitation using the maximum likelihood EM reconstruction algorithm," IEEE Trans. Med. Imaging, vol. 13, no. 3, pp. 526-537, Sept. 1994.

[38] J. A. Fessler, "Mean and variance of implicitly defined biased estimators (such as penalized maximum likelihood): Applications to tomography," IEEE Trans. Image Processing, vol. 5, no. 3, pp. 493-506, Mar. 1996.

[39] K. Lange and R. Carson, "EM reconstruction algorithms for emission and transmission tomography," J. Comput. Assisted Tomog., vol. 8, no. 2, pp. 306-316, Apr. 1984.

[40] N. H. Clinthorne, T. S. Pan, P. C. Chiao, W. L. Rogers, and J. A. Stamos, "Preconditioning methods for improved convergence rates in iterative reconstructions," IEEE Trans. Med. Imaging, vol. 12, no. 1, pp. 78-83, Mar. 1993.

[41] L. T. Chang, "A method for attenuation correction in radionuclide computed tomography," IEEE Trans. Nucl. Sci., vol. 25, no. 1, pp. 638-643, Feb. 1978

[42] H. H. Barrett, J. L. Denny, R. F. Wagner, and K. J. Myers, "Objective assessment of image quality, II: Fisher information, Fourier crosstalk, and figures of merit for task performance," J. Opt. Soc. Amer. Ser. A, vol. 12 , no. 5, pp. 834-52, May 1995.

[43] S. R. Meikle, M. Dahlbom, and S. R. Cherry, "Attenuation correction using count-limited transmission data in positron emission tomography," J. Nucl. Med., vol. 34, no. 1, pp. 143-150, Jan. 1993.

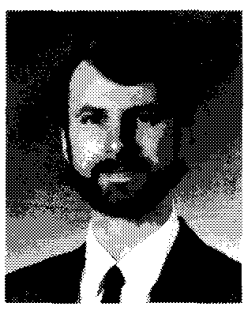

Jeffrey A. Fessler (S'83-M'90) received the B.S.E.E. degree from Purdue University in 1985 , the M.S.E.E. degree from Stanford University in 1986, and the M.S. degree in statistics from Stanford University, Stanford, CA, in 1989. From 1985 to 1988 , he was a National Science Foundation Graduate Fellow at Stanford, where he received the Ph.D. degree in electrical engineering in 1990.

Since 1990, he has been with the University of Michigan, Ann Arbor. From 1991 to 1992, he was a Department of Energy Alexander Hollaender Post-Doctoral Fellow in the Division of Nuclear Medicine at the University of Michigan. From 1993 to 1995, he was an Assistant Professor in Nuclear Medicine and the Bioengineering Program. Since 1995, he has been with the Department of Electrical Engineering and Computer Science. His research interests are in statistical aspects of imaging problems.

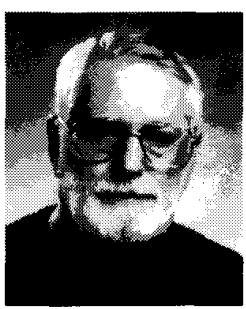

W. Leslie Rogers (M'83) received the Ph.D. degree in nuclear physics from Case Institute of Technology, Cleveland, $\mathrm{OH}$, in 1967

He joined Bendix Aerospace Corporation, Ann Arbor, MI, in 1968, where he worked on designs for the Mars Viking life detection experiment and instrumentation for gamma-ray imaging in nuclear medicine. He joined the University of Michigan in 1970, where he currently holds an appointment as Professor and Research Scientist in the Division of Nuclear Medicine, Department of Medicine, University of Michigan. He is also a member of the Bioengineering Faculty there. His research interests are in the area of nuclear medical imaging instrumentation and quantitative imaging.

Dr. Rogers is a member of the NIH Diagnostic Radiology Study Section and the Society of Nuclear Medicine, and is an AIMBE Fellow. 